\title{
Diacronie
}

Studi di Storia Contemporanea

$\mathrm{N}^{\circ} 32,4 \mid 2017$

Proiezioni individuali e agire collettivo nella storia

\section{Emanuela Locci, Storia della massoneria femminile. Dalle corporazioni alle obbedienze}

\section{Luca Giuseppe Manenti}

\section{(2) OpenEdition}

Edizione digitale

URL: http://journals.openedition.org/diacronie/6962

DOI: 10.4000/diacronie.6962

ISSN: 2038-0925

Editore

Association culturelle Diacronie

Notizia bibliografica digitale

Luca Giuseppe Manenti, « Emanuela Locci, Storia della massoneria femminile. Dalle corporazioni alle obbedienze », Diacronie [Online], № 32, 4 | 2017, documento 13, Messo online il 29 décembre 2017, consultato il 24 septembre 2020. URL : http://journals.openedition.org/diacronie/6962 ; DOI : https:// doi.org/10.4000/diacronie.6962 


\title{
Diacronie
}

Studi di Storia Contemporanea

\section{$32,4 / 2017$}

Proiezioni individuali e agire collettivo nella storia. Ruoli sociali, aspetti politici e nodi storiografici tra pubblico e privato.

\section{RECENSIONE: Emanuela LOCCI, Storia della massoneria femminile. Dalle corporazioni alle obbedienze, Roma, Bastogi, 2017, 167 pp.}

\author{
A cura di Luca G. MANENTI
}

Per citare questo articolo:

MANENTI, Luca G., «RECENSIONE: Emanuela LOCCI, Storia della massoneria femminile. Dalle corporazioni alle obbedienze, Roma, Bastogi, 2017, 167 pp.», Diacronie. Studi di Storia Contemporanea : Proiezioni individuali e agire collettivo nella storia. Ruoli sociali, aspetti politici e nodi storiografici tra pubblico e privato, 32, 4/2017, 29/12/2017.

URL: < http://www.studistorici.com/2017/12/29/manenti_numero_32/>

Diacronie Studi di Storia Contemporanea $\rightarrow$ http://www.diacronie.it Rivista storica online. Uscita trimestrale.

redazione.diacronie@hotmail.it

Comitato di direzione: Naor Ben-Yehoyada - João Fábio Bertonha - Christopher Denis-Delacour - Maximiliano Fuentes Codera Anders Granås Kjøstvedt - John Paul Newman - Deborah Paci - Niccolò Pianciola - Spyridon Ploumidis - Wilko Graf Von Hardenberg

Comitato di redazione: Jacopo Bassi - Luca Bufarale - Gianluca Canè - Fausto Pietrancosta - Alessandro Salvador - Matteo Tomasoni - Luca Zuccolo 


\title{
13/ Emanuela LOCCI, Storia della massoneria femminile. Dalle corporazioni alle obbedienze, Roma, Bastogi, 2017, 167 pp.
}

\author{
A cura di Luca G. MANENTI
}

Nel panorama storiografico italiano il tema del rapporto tra donne e libera muratoria è stato vittima di un tendenziale disinteresse ${ }^{1}$, causato, secondo Emanuela Locci, dalla ritrosia degli studiosi a occuparsi di soggetti inutili ai fini carrieristici: «gli storici tendono a trascurare argomenti che possono sembrare marginali, di nicchia, che poi non sono proficui dal punto di vista accademico» ${ }^{2}$. Una pecca cui l'autrice fa ammenda con un lavoro che ripercorre con scrupolo nascita ed evoluzione dei maggiori sodalizi femminili massonici e para-massonici europei, scandito in otto capitoli che spaziano dal problema dell'iniziazione delle donne alla storia dei circuiti liberomuratori disposti e meno disposti a riceverle, passando per il variegato sottobosco di società androgine, logge d'adozione o a tinta unita, formate da aristocratiche e alto-borghesi.

Una ricerca basata su fonti scritte e in minor misura iconografiche; è mancato invece, evidentemente per ragioni di spazio, uno scavo nel variegato universo della musica e dei canti massonici, che pure avrebbe potuto fornire qualche dato interessante ${ }^{3}$. L'analisi parte dalle gilde medievali, cui le donne parteciparono in posizione subordinata, così come subordinata rimase a lungo la loro presenza nella successiva massoneria speculativa, salvo eccezioni significative. Tre sono le aree geografiche su cui Locci si concentra: Inghilterra, Francia e Italia; ognuna contraddistinta da atteggiamenti particolari e opinioni diverse sull'ipotesi dell'accettazione in loggia delle estromesse.

\footnotetext{
${ }^{1}$ Sulla massoneria femminile, la cui scarsa attrattiva esercitata sulla storiografia nostrana è testimoniata dai pochi titoli in lingua italiana che compaiono in appendice al volume qui recensito, si veda da ultimo il seguente articolo, non citato da Locci perché troppo recente: MOLA, Aldo Alessandro, «L'Oriente è rosa. La massoneria femminile», in Il Giornale del Piemonte e della Liguria, 29 ottobre 2017, pp. 1, 11.

${ }^{2}$ LOCCI Emanuela, Storia della massoneria femminile. Dalle corporazioni alle obbedienze, Roma, Bastogi, 2017, p. 15.

${ }^{3}$ Si veda per esempio una strofa del coro che gli apprendisti di una loggia italiana fondata nel 1751 cantavano durante il banchetto, chiarificatrice del motivo per cui le donne non vi erano ammesse: «Se tra noi luogo non hanno/ Le tue ninfe, Amor, perdona;/ Che ove il nome tuo risuona,/ Tutto è colpa e tutto è inganno/ Né tener san donne imbelli/ Il segreto dei fratelli»; citata in FRANCOVICH, Carlo, Storia della massoneria italiana. Dalle origini alla Rivoluzione francese, Firenze, La Nuova Italia, 1974, p. 106.
} 
L'Inghilterra è stata la culla della massoneria moderna e il centro d'elaborazione del documento che ha fatto dell'esclusivismo maschile l'inderogabile regola vigente nel tempio: le Costituzioni di Anderson del 1723, che nell'elenco dei non ammissibili annoveravano servi, donne e moralmente indegni. Agli inizi del Novecento sorsero nell'isola delle officine femminili, rimaste allo stadio di organismi irregolari per il mancato riconoscimento da parte della Gran Loggia d'Inghilterra, che solo da ultimo è giunta a instaurare con le sorelle, senza mutare la sostanza della propria impostazione, cordiali rapporti collaborativi. Di ogni esperienza latomistica l'autrice fornisce in abbondanza nomi, riti, cariche, organigrammi, ampliamenti e scissioni, riportando talvolta in modo minuzioso decori e colori delle logge in determinate tornate; talaltra i dialoghi tra chi iniziava e chi veniva iniziata, rendendo possibile desumere gli obiettivi e gli intendimenti delle mopse ${ }^{4}$. Significativo il seguente scambio di battute: «Domanda: Quale è il vostro scopo entrando a far parte della Massoneria? Risposta: Il mio scopo è quello di mettere fine al pregiudizio che ne esclude le donne, poiché nutro serie speranze che, grazie alla loro ammissione, si potrà compiere all'interno delle logge un'opera di risveglio delle coscienze» ${ }^{5}$.

Utili poi i profili biografici dei protagonisti e delle protagoniste della trama dipanata, solitamente collocati in note a piè di pagine dense di dati. Ricorrente, ma non stupefacente, il fatto che le donne desiderose di seguire il cammino iniziatico siano spesso state figlie o mogli di massoni, la cui vicinanza aveva stimolato una vocazione tramutatasi, per le più caparbie, nella faticosa entrata nell'istituzione in ruoli di comprimarie. Interessante lo studio svolto sugli intrecci tra massoneria e società teosofica, dove le donne hanno giocato la parte del leone, spingendo per la moltiplicazione di logge miste nelle colonie dell'impero britannico.

È stata però la Francia il luogo più fecondo per la crescita della massoneria femminile. Nel 1893 fece d'apripista Le Droit Humain, obbedienza avente lo scopo, come il titolo stesso dell'associazione denunciava, d'estendere i diritti a tutti, evitando distinzioni di genere. Tra i meriti dell'autrice vi sono da un lato quello d'aver saputo lumeggiare i perché dell'impraticabilità di una massoneria femminile - si veda l'osservazione sull'idea cristiana di una donna ritenuta intrinsecamente inabile a "entrare in contatto con il divino, rimanendo confinata alla sua dimensione materiale» ${ }^{6}$ - dall'altro di aver colto i parallelismi tra le situazioni nella vita civile e nelle officine: «mentre si assisteva al progredire, non senza contraddizioni, della condizione femminile dal punto di vista sociale, lavorativo ed economico, vi era un fermento anche per quel che concerne il sistema massonico e la presenza attiva delle donne in massoneria» ${ }^{7}$. La libera muratoria andrebbe pertanto osservata - implicitamente e correttamente afferma l'autrice - non come un elemento

\footnotetext{
${ }^{4}$ Il termine indica i membri femminili delle logge miste, derivato, scrive Heckethorn, «from the German word Mops, a young mastiff, the symbol of fidelity»: HECKETHORN, Charles William, The Secret Societies Of All Ages And Countries, vol. II, London, George Redway, 1897, p. 85.

${ }^{5}$ LOCCI Emanuela, Storia della massoneria femminile, cit., p. 67.

${ }^{6}$ Ibidem, p. 39.

${ }^{7}$ Ibidem, p. 66.
} 
avulso dalla società, ma quale cartina di tornasole per cogliere tendenze e orientamenti generali.

In Italia la creazione di logge femminili è stata precoce, risalente alla Napoli di fine XVIII secolo, ma strada facendo la questione della loro liceità ha risentito delle complicate relazioni tra le varie obbedienze sparse sul territorio e dello scontro ottocentesco tra correnti progressiste, rappresentante da Camillo Finocchiaro Aprile, e conservatrici, incarnate da Ludovico Frapolli. Forte $\mathrm{fu}$ il sostegno prestato alle prime dal massone Giuseppe Garibaldi, che patrocinò l'iniziazione della figlia Teresita. L'autrice riassume il complesso quadro delle prese di posizione pro e contro ${ }^{8}$ svelandone le motivazioni nascoste, portando in superficie il sostrato di timori e incertezze che caratterizzavano la natura del rifiuto dell'iscrizione di elementi potenzialmente perturbatori di usanze e gerarchie costituite: «Le logge [femminili] si proponevano non solo come punto di incontro tra esponenti della nobiltà che operavano a scopi filantropici o mondani, ma erano il vero centro delle rivendicazioni femminili sull'emancipazione della donna» ${ }^{9}$.

Oggi il Grande Oriente d'Italia, la più numerosa famiglia massonica della penisola, vieta l'iscrizione delle donne, accettate invece nella Gran Loggia d'Italia, ma è altresì attiva dal 1990 una Grande Loggia Massonica Femminile d'Italia, attualmente l'unica obbedienza di questo tipo riconosciuta all'estero ${ }^{10}$.

La monografia di Locci risulta, in definitiva, ben svolta, aggiornata, istruttiva, tanto che volentieri le si perdona, qua e là, un'eccessiva fiducia nelle fonti. Ad esempio, i racconti riguardanti signore scoperte a sbirciare di nascosto le sedute dei fratelli e perciò ammesse in loggia per evitare uno scandalo, andrebbero interpretati non solo - o non tanto - in senso letterale, come fatti di cui è pure possibile, come legittimamente fa l'autrice, individuare un nucleo di verisimiglianza, ma anche - o a maggior ragione, data la sospetta ciclicità con cui ritornano identici in zone e tempi distanti - alla stregua di mitologemi, atti a giustificare, tramite un collaudato espediente retorico, l'improvvida licenza a varcare le soglie del tempio concessa alle curiose dalla controparte maschile (col paradosso che i vizi femminili presi a scusa per la selezione operata in loggia, ossia la supposta incapacità di mantenere un segreto e la propensione all'intrigo $^{11}$, si siano poi rivelati la chiave per l'abbattimento di ogni restrizione). Un discorso

\footnotetext{
${ }^{8}$ Un quadro troppo ampio per essere restituito nella sua interezza, per cui è stato inevitabilmente sacrificato da Locci il pensiero di un massone importante come Francesco De Luca, Gran maestro del Grande Oriente d'Italia fra il 1865 e il 1867 e, negli stessi anni, vicepresidente della Camera, il quale dimostrò attenzione alla questione della massoneria femminile pur non spostandosi da posizioni tradizionali. Coinvolgendole nei lavori massoni, secondo De Luca, le donne «verrebbero facilmente a screditare colla loro leggerezza la più pura delle istituzioni. Però non si perda di vista la questione, e la si studi onde arrivare allo scopo [della loro ammissione]»: D'ALESSANDRI, Antonio, «Recensione a POLO FRIZ, Luigi, MAMMONE, Domenico, Francesco De Luca. Rivoluzionario, Deputato, Gran Maestro della Massoneria, Cosenza, Editore Brenner, 2003», in Hiram, 4/2004, pp. 110-112.

${ }^{9}$ LOCCI Emanuela, Storia della massoneria femminile, cit., p. 109.

${ }^{10}$ Tutte e tre le obbedienze citate dispongono di siti ben curati, testimonianza di una volontà di proporsi come attori sociali trasparenti e attivi sul palcoscenico mediatico URL: $<$ http//www.grandeoriente.it/>; $<$ http://www.granloggia.it/ >, < http://www.granloggiafemminile.it/ > [consultati il 20 novembre 2017].

${ }^{11}$ RÓBERT, Péter, "The Fair Sex" in a "Male Sect": Gendering the Role of Women in Eighteenth-Century English
} 
simile vale per l'aneddoto della partecipazione, data per vera da Locci, di Helena Blavatsky alla guerre garibaldine, evenienza accreditata dalla stessa teosofa e poi ripresa da uno stuolo di seguaci, ma priva di reali riscontri e giudicata «implausibile» dai migliori esperti d'esoterismo ${ }^{12}$.

Nonostante queste piccole sbavature, che non inficiano oltremodo il valore di un elaborato ricco di informazioni pazientemente raccolte, il volume rimane un vademecum attendibile per chi voglia approcciarsi a una materia insieme spinosa e affascinante. L'apparato finale contemplante bibliografia, indici archivistici, elenchi di articoli e sitografia, costituisce una sorta di piccolo saggio nel saggio: uno strumento d'estrema comodità per avventurarsi nel ginepraio delle pubblicazioni di settore.

In coda al testo l'autrice abbraccia in prima persona una causa che ha il sapore di una giusta battaglia per la parità dei sessi, auspicando il pieno superamento degli impedimenti che ancora sussistono all'accesso delle donne in officina:

Nel corso del tempo sono state fornite dagli ambienti massonici altre giustificazioni per spiegare la negazione dell'ammissione delle donne nei templi. In alcuni casi si è parlato di Iniziazione: che tipo di iniziazione dare alle donne? Che rituali seguire? Senza voler esprimere in questa sede un'opinione in tal senso, non è compito demandato allo storico, pare che questi ostacoli possano essere facilmente superati, oggi più che mai ${ }^{13}$.

La speranza è che Locci prosegua il sentiero intrapreso, tappa di una produzione saggistica già consistente, regalandoci nuove analisi sulla massoneria femminile in altri contesti statali, allargando le conoscenze sul tema per il pubblico di lingua italiana.

Freemasonry, in CROSS, Marie (ed.), Gender and Fraternal Orders in Europe from 1200 until Present, Basingstoke, Palgrave Macmillan, 2010, pp. 133-155, p. 136.

${ }^{12}$ PASI, Marco, Teosofia e antroposofia nell'Italia del primo Novecento, in CAZZANIGA Gian Mario (a cura di), Storia d'Italia, annali 24, Esoterismo, Torino, Einaudi, 2010, pp. 569-598, p. 577 nota 19.

${ }^{13}$ LOCCI Emanuela, Storia della massoneria femminile, cit., p. 141. 


\section{L'AUTORE}

Luca G. MANENTI, laureato in Storia a Milano, è dottore di ricerca e cultore della materia in Storia contemporanea presso l'Università degli Studi di Trieste. Si occupa d'associazionismo patriottico, culture ebraica e armena, massoneria, irredentismo e nazionalismo tra Risorgimento e Grande guerra. Ha pubblicato vari saggi in riviste e volumi collettanei e le monografie: Massoneria e irredentismo (Trieste, IRSML, 2015), Da Costantinopoli a Trieste (Milano, Biblion, 2015).

URL: < http://www.studistorici.com/progett/autori/\#Manenti > 